\title{
Influence of fading duration on TCATA evaluation
}

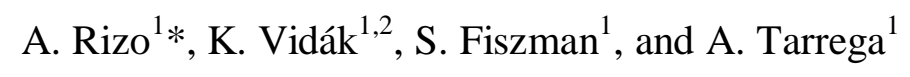

${ }^{1}$ Instituto de Agroquímica y Tecnología de Alimentos (IATA-CSIC). Agustín Escardino, 7. Paterna (Valencia), Spain

${ }^{2}$ Faculty of Food Science, Szent István University, Villányi út 29-43, H-1118 Budapest, Hungary

*Corresponding author's email: arantxa.rizo@iata.csic.es

Key words: Dynamic sensory evaluation, fading, discrimination, TCATA curves, sensory trajectories 


\begin{abstract}
Temporal Check All that Apply (TCATA) is a dynamic sensory evaluation technique in which participants select the terms they consider apply at each moment from a list of attributes and deselect them when they no longer apply. TCATA Fading is a variant that facilitates the TCATA task by making term deselection automatic and progressive over a period of a few seconds. The aim of the present work was to assess the influence of fading duration on the TCATA Fading task and results. Three studies evaluated two fading durations (4 s vs $8 \mathrm{~s}$ in Study 1 and 2, and $3 \mathrm{~s}$ vs $6 \mathrm{~s}$ in Study 3), using commercial cooked ham, a strawberry-flavoured dessert and orange juice, and compared task performance, TCATA curves and sensory trajectories. Longer fading times resulted in TCATA curves with higher citation proportions, in which differences among samples lasted slightly longer. TCATA curves and trajectories provided very similar information on differences in dynamic sensory perception of the products. Fading duration seems to be not critical when using values within a "reasonable" range, which can be determined in preliminary essays. Ideally it would be advisable to choose the shorter fading time in the range to avoid possible overestimation of the duration of sensations and "filling gaps" procedure to compensate the increase in gap events due to delay in reselection. This is the first paper to show the implications of duration of fading in TCATA evaluation.
\end{abstract}




\section{Introduction}

In the last decade, interest in evaluating the temporal aspects of sensory perception has grown, as they can provide a more realistic or complete picture of the sensations elicited by food products (Di Monaco, Su, Masi, \& Cavella, 2014). Different dynamic sensory methods have been developed to provide insights into the sequence in which in-mouth sensations occur during consumption. This is relevant for many products, especially those with complex matrices such as wine (Meillon, Urbano \& Schlich, 2009; Sokolowsky \& Fischer, 2012), ice cream (Varela, Pintor \& Fiszman, 2014) or dairy products (Pineau et al., 2009).

The temporal sensory method most often applied until now has been Temporal Dominance of Sensations (TDS), which was originally proposed as a multi-attribute method that scaled the intensities of a sequence of dominant attributes (Pineau, Cordelle $\&$ Slich, 2003). Nowadays, the TDS test is based just on selecting, from a list of terms, the dominant sensation or the one that caught the assessor's attention at each moment during consumption of the product (Di Monaco et al., 2014; Schlich, 2017). New variants of the original TDS have been proposed, such as TDS by modalities (M-TDS) in which sensations of texture and flavour are evaluated separately (Nguyen, Næs \& Varela, 2018) or temporal drivers of liking (TDL) (Thomas, Visalli, Cordelle \& Schlich, 2015) which uses TDS in conjunction with consumer acceptance.

More recently, another multi-attribute temporal method called Temporal Check all that apply (TCATA) has been introduced by Castura, Antúnez, Giménez, and Ares (2016). TCATA is a temporal extension of Check all that apply (CATA) in which assessors are given a list of attributes from which they select all the terms they consider apply at each point in the evaluation time and deselect them when they no longer apply. Unlike TDS, TCATA is not based on the concept of dominance, so several attributes can be selected at the same time. Up to now, different studies have applied TCATA to a wide range of food products, such as bread, salami, pategrás cheese, strawberry yogurts, milk desserts, orange juice, marinated mussels, milk desserts, mint chocolate, and hard cheese (Alcaire et al., 2017; Ares et al., 2015; Ares et al., 2016; Castura et al., 2016), wine (Baker, Castura, \& Ross, 2016), probiotic chocolate flavoured milk (Oliveira, Antúnez, Giménez, Castura, Deliza, \& Ares, 2015), milk chocolate (Ares, Alcaire, Antúnez, Vidal, Giménez, \& Castura, 2017), fermented milks (Esmerino et al., 2017), dried 
apricots and peanuts (Jaeger, Beresford, Hunter, Alcaire, Castura, \& Ares, 2017), sparkling wines (McMahon, Culver, Castura, \& Ross, 2017), canned pineapple and crackers (Jaeger, Alcaire, Hunter, Jin, Castura, \& Ares, 2018), yoghurts with oat flakes (Nguyen et al., 2018), cooked ham (Rizo, Peña, Alarcon-Rojo, Fiszman, \& Tarrega, 2018), beer (Ramsey et al., 2018), and other products apart from food, such as cosmetic emulsions (Boinbaser, Parente, Castura, \& Ares, 2015). Both TDS and TCATA have proven to be useful sensory methodologies that can be used both with trained assessors and with consumers to supply valuable information to describe the evolution of sensory perception throughout consumption, in some cases discriminating better between samples than other conventional sensory profiling methods (Ares et al., 2015; Di Monaco et al., 2014).

One of the drawbacks of the TCATA method is that assessors may encounter difficulties in the evaluation, as they have to pay attention to two tasks simultaneously (selection and deselection of attributes). Higher citation proportions have been detected at the end of the evaluation (Castura et al., 2016), which suggests that when using TCATA the assessors tend to focus more on continuously selecting than on deselecting terms and some attributes could remain selected even when they no longer apply (Ares et al., 2016). For this reason, a new version of TCATA called TCATA Fading has recently been proposed by Ares et al. (2016). In the TCATA Fading variant, term deselection is automatic and progressive over a pre-defined duration of few seconds, so when participants still perceive a term after it has been deselected automatically they have to select it again. Automatic deselection of terms has been proved to facilitate the assessor's task (Ares et al., 2016). Therefore, TCATA Fading seems to provide a more accurate description of the sensory dynamics of products during consumption and to improve sample discrimination compared with TCATA (Ares et al., 2016). Previous studies have shown the relevance to TCATA and TCATA Fading results of different methodological aspects such as the number of attributes presented to assessors (Jaeger et al., 2018), or the use of a previous familiarization step before the task (Jaeger et al., 2017). Up to now, TCATA Fading has only been used in few studies of different solid food products (Ares et al., 2016; Jaeger et al., 2018). For all these studies, the predefined duration of fading was $8 \mathrm{~s}$, selected by the different authors as the most appropriate period of time to facilitate the assessor's task. However, the same authors have highlighted the need for further methodological studies to discover how to define the duration of fading in TCATA studies, and have already suggested the potential 
relevance of different elements of the task such as the duration of evaluation, the characteristics of the dynamic profile of the product and the number of terms. To the best of the present authors' knowledge, there has been no research on the influence of fading duration on the TCATA evaluation.

Therefore, the aim of the present work was to ascertain whether the length of fading time affects how assessors perform the TCATA task, and the results.

\section{Materials and methods}

\subsection{Samples}

Three different types of samples were selected for the study. A solid (cooked ham), a semi-solid (a strawberry dessert) and a liquid product (orange juice). All the samples were commercial products available on the Spanish market.

In Study 1, five commercial cooked ham products (HamA-HamE) with different percentages of pork meat and salt were studied. Oral processing of these same samples and its relation to the dynamics of sensory perception have already been studied and discussed in Rizo et al. (2018). HamA was a cooked pork luncheon meat with 55\% pork, whereas the rest of the cooked ham products contained at least $82 \%$ pork. The label of sample HamE included the claim "reduced salt content" and according to labels, this ham contained $21 \%$ to $40 \%$ less salt than the rest of the samples. For the evaluation, cooked ham slices $(2-\mathrm{mm}$ thick) were cut into rectangular pieces $(40 \times 100 \mathrm{~mm}$, weight about $7 \pm 1 \mathrm{~g}$ ) and rolled up to make ham rolls (40mm length) that were served to the assessors at $4-6^{\circ} \mathrm{C}$.

In Study 2, four commercial strawberry-flavoured fresh cheese desserts (petit suisse style) were chosen. DessA and DessC corresponded to the regular version of two brands of the product and DessB and DessD were the high-protein version of the product for the same two brands. The regular version products contained $2.5-2.6 \%$ fat and $6 \%$ protein while the high protein versions contained $0 \%$ fat and $8.5-10 \%$ protein. The samples were purchased and stored at $4^{\circ} \mathrm{C}$. The serving size was the equivalent of a teaspoon $(10 \pm 1 \mathrm{~g})$ and was presented to the participants in $100 \mathrm{~mL}$ plastic cups at a temperature of $7-10^{\circ} \mathrm{C}$.

The samples in Study 3 were four commercial orange drinks. Two were the regular version of the product (OrangA and OrangC) and the other two (OrangB and OrangD) were the "no added sugar" versions. OrangA and OrangC included sucrose among their 
ingredients, while OrangB and OrangD included sweeteners (sucralose and acesulfame $\mathrm{K}$ respectively). The serving size corresponded to one sip $(15 \pm 1 \mathrm{~g})$, presented in 100 $\mathrm{mL}$ plastic cups at a temperature of $10-14^{\circ} \mathrm{C}$.

In all three studies, the samples were identified with random three-digit codes.

\subsection{Assessors}

Assessors for the three studies were recruited among students and employees of the Institute of Agrochemistry and Food Technology (IATA-CSIC).

In Study 1, the seventeen assessors who took part in the evaluation had no previous experience with TCATA but did have experience with sensory descriptive evaluation, attribute recognition and scaling in other categories of products. Prior to the TCATA tests, the assessors participated in 3 training sessions for recognition and agreement on the sensory terms presented in the TCATA task. In the first session, a list of nine attributes (previously obtained in a vocabulary generation session with six assessors) and their descriptions was proposed to the assessors. Different ham samples were provided for tasting. After an open discussion among the participants, a final list of seven attributes and their descriptions was reached by consensus. The second and third training sessions focused on recognition of each attribute in different cooked ham samples, using paired comparison tests. At the end of each session, open discussions were conducted to check on the agreements and disagreements concerning the evaluation of each attribute. A fourth session was devoted to familiarizing the assessors with the TCATA Fading task and software use.

In Studies 2 and 3, the evaluation was carried out by untrained consumers: 68 consumers of strawberry-flavoured fresh cheese desserts (56\% women, 18 to 65 years old) participated in Study 2 and 70 consumers of orange juice (57\% women, 18 to 65 years old) in Study 3 (Table 1).

\subsection{Sensory evaluation procedure}

In all three studies, sensory evaluation of the samples was carried out using the TCATA Fading technique, employing two different fading times. For each study, preliminary trials with 6-8 assessors were conducted to determine the length of the evaluation and the range of fading times that would be adequate for the assessors to complete the task of selecting and re-selecting terms. Accordingly, the two fading durations chosen were 4 $\mathrm{s}$ and $8 \mathrm{~s}$ for Studies 1 and 2. For Study 3 fading durations were $3 \mathrm{~s}$ and $6 \mathrm{~s}$. 
The TCATA attribute list in Study 1 was obtained from the training sessions. For Studies 2 and 3, the lists of sensory attributes were those obtained in preliminary trials with 6 to 8 assessors, who were asked firstly to indicate the attributes that differed among samples and secondly to indicate the attributes they perceived while consuming each sample. The attributes they cited most were selected for inclusion in the list. The terms were related to both flavour and texture, except in Study 3, where they were only related to flavour (Table 1). Seven terms were used in Study 1, nine terms in Study 2, and five terms in Study 3. The tests were conducted in Spanish and the terms presented in this paper have been translated into English. Before the evaluation, the assessors were instructed in how to carry it out. They were asked to read the terms before starting the test in order to locate them on the screen. To begin the test, they were instructed to take a whole roll/spoon/sip and simultaneously click on the "start" button to start the sample evaluation immediately. During evaluation, they were asked to select all the terms that described the sensations they perceived at each moment of the evaluation and to click the "stop" button when they did not perceive any further sensation. As a general rule, they were told that at each moment of the evaluation they had to check that all the attributes they perceived were highlighted on the screen, as the terms would be deselected automatically.

The evaluation lasted until the sensory sensations ceased and they pushed the stop button. A break of $20 \mathrm{~s}$ between samples was established to clean the palate with still mineral water, except in Study 3 (orange juice), when the break was $60 \mathrm{~s}$. For all three studies, the participants used a mouse to complete the task.

Consumers in studies 2 and 3 were first informed about the evaluation procedure (as indicated above) then received a first sample as a trial to familiarize themselves with the test procedure. They were informed that this was just a trial and were encouraged to ask questions and raise any doubts. After the trial run, they were asked to evaluate five samples. The first of the five was a dummy sample (though the participants were not aware of this) and the results were discarded. For all the consumers and sessions, the trial samples and dummy samples were two of those studied (DessB and DessC for Study2; OrangA and Orang D for Study 3).

In Study 1, the semi-trained assessors performed three replicate evaluations per fading time, totalling six different sessions. In Studies 2 and 3 the consumers participated in two evaluation sessions, one per fading time, on two different days. The order in which the assessors completed the two evaluations (short fading and long fading) was 
balanced across assessors. Half started with the short fading duration TCATA session and the other half with the long fading duration session. The order of sample presentation and the order of attributes in the TCATA list were varied among the assessors, following a Williams Latin square design, but for each assessor the list of attributes remained the same for all the samples. Sensory evaluation took place in standard sensory booths designed in accordance with ISO 8589 (ISO, 2007), under artificial daylight and temperature control $\left(22{ }^{\circ} \mathrm{C}\right)$. The data collection and analysis were carried out using Compusense Cloud (Compusense Inc., Guelph, Canada).

\subsection{Data analysis}

\subsubsection{Facts during task completion}

The individual data registered per attribute, sample, session and assessor were analysed to determine whether there were differences in how the assessors performed the TCATA task over the different fading durations. The total evaluation time, number of times an attribute was selected, total time each attribute remained selected, and gaps and overlaps during evaluation were calculated and analysed. To study the number of times that an attribute was selected during the same run and the time that each attribute remained selected, only the cases in which the attribute was selected were considered.

When successive selections of the same attribute occurred in the same run, two phenomena were observed: gaps and overlaps. Gaps were identified as periods of time between successive selections in which an attribute was not selected to describe a sample.

In many cases, the assessor selected the same attribute again when it was still selected and fading. These were termed "overlaps". In the Compusense TCATA test, when this happens the first selection stops, so it lasts for a shorter time than the set fading period, while the second selection starts at exactly the same second as the first selection finishes and continues for the complete fading time established unless a new selection stops it earlier, thus causing a new overlap.

Two factor ANOVA with interaction was used to evaluate the effects of sample and fading time on the duration of attribute selection and the number of selections. These analyses were performed with XLSTAT statistical software (version 2016, Addinsoft, Paris, France).

\subsubsection{TCATA curves and trajectories}


To obtain the TCATA curves, the procedures proposed by Castura et al. (2016) were followed.

The number of terms selected by each assessor for each of the samples at each moment of the evaluation (every $1 \mathrm{~s}$ ) was obtained. For each sample, the data were aggregated across all the participants to obtain line plots. The citation proportion represented by the TCATA curves for each attribute was calculated as the proportion of cases (assessors $x$ replicates) selected to describe a sample at a given time (every $1 \mathrm{~s}$ ) of the evaluation. The TCATA curves were smoothed using a spline-type polynomial. The Fisher-Irwin test (Fisher, 1935; Irwin, 1935) was used to evaluate the significance of the differences in attribute citation proportions between each sample relative to the average of the rest of the samples from each fading time experiment. When the citation proportions of one sample differed significantly from the average for the other products, the differences were highlighted at that time segment on the TCATA curves. Comparisons were made through a two-sided test at a level of $5 \%$.

To study the influence of fading duration, the differences in citation proportion between the two fading times (4 and $8 \mathrm{~s}$ for Study 1 and 2, and $3 \mathrm{~s}$ and $6 \mathrm{~s}$ for Study 3) were calculated. A sign test was applied at each time point for each attribute to evaluate whether the citation proportions for the same sample with a different fading time were statistically different from zero at the 5\% significance level. Data analysis was carried out using R software version 3.3.2 with $\mathrm{R}$ package tempR (v. 0.9.9.10) (Castura, 2016). The TCATA trajectories were also obtained through the procedure described in Castura et al. (2016).

Correspondence analysis using $\chi^{2}$ distance was applied to the citation proportions of the attributes (columns) for each sample for specific time slices of 5 seconds (rows). The data from the short and long fading tests were included in the same analysis for comparison. Asymmetric row plots were used to show the TCATA trajectories. These analyses were carried out using XLSTAT statistical software (version 2016, Addinsoft, Paris, France).

\section{Results}

\subsection{Facts during task completion}


The total duration time that corresponded to the time when assessors pushed the stop button varied significantly with fading duration in the three studies $(\mathrm{p}<0.001, \mathrm{p}<0.001$ and $\mathrm{p}=0.024$ for Studies 1,2 and 3, respectively). The longer the fading time, the longer the total duration of evaluation. The average total evaluation times were $31.9 \mathrm{vs}$ $34.8 \mathrm{~s}$ in Study 1, 22.3 vs $25.5 \mathrm{~s}$ in Study 2 and 20.5 vs $22.3 \mathrm{~s}$ in Study 3 (short fading vs long fading tests). The number of different attributes selected in each evaluation did not vary significantly with fading time but did vary between the studies: 3.5 vs 3.5 in Study 1, 4.5 vs 4.6 in Study 2 and 3.1 vs 3.1 in Study 3 for the short and long fading times respectively. The number of total selections (clicks) that the participants made during each evaluation varied slightly with fading time: 9.3 vs 8.3 for Study $1,8.4$ vs 7.6 for Study 2 and 8.2 vs 7.6 for Study 3, for the short and long fading times respectively.

Table 2 shows the average total time each attribute remained selected and the number of selections over the same run. According to ANOVA results, no significant interaction effects (fading time $\mathrm{x}$ sample) were found indicating that fading time effect did not significantly depend on sample. In all three studies, the duration of attribute selection was significantly higher in the long fading tests than in the tests with short fading. In contrast, the number of times an attribute was selected over a run did not differ significantly between the short and long fading tests in any of the studies, except for the attribute consistent in Study 2, which showed a slightly higher number of selections (1.9) in the short fading test than in the long fading test $(1.4)(p=0.04)$.

For each attribute, the percentage of cases in which it was reselected out of the total cases in which it was selected is shown in Table 3. The percentage of reselection varied considerably among the studies but the differences between the short and long fading time tests were small and did not follow a clear pattern. In most cases, gaps appeared when reselection occurred. According to Vidal et al. (2017), gaps of short duration can be due to an assessors' delay in reselecting a term that was still applicable after the selection had faded. The duration of each gap was calculated. On observing the distribution of gap durations, most gaps (55-73\%) were shorter than $4 \mathrm{~s}$ in all three studies. Gaps of less than 2 seconds were the most frequent, comprising $32.0 \%$ and $38.1 \%$ (Study 1), 38.8\% and $42.7 \%$ (Study 2), and 45.8 and $45.3 \%$ (Study 3 ) of the total gaps for the short and long fading times, respectively. Gaps with a duration between 2.0 and 3.9 seconds also showed a high frequency of occurrence $(23.2 \%$ vs $23.6 \%$ for Study $1,21.7 \%$ vs $26.3 \%$ for Study 2, and $25.5 \%$ vs $27.8 \%$ for Study 3, again for the short and 
long fading times respectively). The frequency of $\geq 4 \mathrm{~s}$ gap durations decreased drastically and included gaps of many different lengths (from 4 to 15 s), each accounting for under $10 \%$ of the total gaps. It was assumed that the very frequent short gaps $(<4 \mathrm{~s})$ were mostly due to the assessor's delay in reselecting a term. For gaps longer than $4 \mathrm{~s}$, it was difficult to ascertain whether they were delays or just that the sensation had ceased and appeared again. As shown in Table 3, both the percentage of cases with gaps $(<4 \mathrm{~s})$ and the differences between the short and long fading times were study-dependent. In Study 3, the number of gaps was much lower for the long fading test than for the short fading test. In Study 1 and 2, the effect depended on the attribute. For most attributes, the percentage of gaps decreased moderately with increased fading time, with the exception of juicy in Study 1 and mouth coating and smooth in Study 2, which presented fewer gaps with the short fading time.

Overlaps occurred when the participant reselected an attribute that was still selected and fading. For each case, the time of fading at which the assessors reselected the attribute was calculated. The distributions of these data for each study are shown in Supplementary data, Fig. 1. In general, few reselections occurred during the first second of fading but after second 2 the frequency of reselections were equally distributed throughout the duration of fading, indicating that in general the participants reselected the term at any time of the fading duration, not only at the end. In the short fading test, but only in Study 1, overlaps seemed to increase in the final seconds of fading. A higher percentage of overlaps was detected in the long fading tests for all attributes in all three studies (Table 3).

\subsection{TCATA curves}

The TCATA plots were built from the citation proportions of the sensory attributes perceived during the consumption time of the cooked ham, strawberry dessert and orange juice samples, using two different fading times. The TCATA curves for all the samples and studies are available to interested readers in Supplementary data, Fig. 2. Figures 1, 2 and 3 show examples of TCATA curves obtained from the short and long fading tests for samples HamA, Dess A and OrangA, corresponding to Studies 1, 2 and 3 , respectively. A thicker line in the curves indicates that for that sample the citation proportion of the attribute was significantly higher than the average citation proportion for the rest of the samples at that evaluation time within the same fading duration 
category. In general, the TCATA curves obtained with the shorter fading time (4 or $3 \mathrm{~s}$ ) and the longer fading time ( 8 or $6 \mathrm{~s}$ ) showed very similar profiles and revealed the same differences among samples.

In Studies 1 and 2, the first sample the consumers evaluated was a trial to familiarize them with the test procedure. After this trial run, they evaluated five samples, the first of which was a dummy sample. In both studies, the TCATA curve obtained from the evaluation of the dummy sample did not differ from the results of the same sample evaluated "formally". This suggests that for untrained assessors conducting TCATA evaluation, the use of a trial sample before evaluation is enough to obtain consistent results and to avoid the first order effect.

\subsubsection{TCATA curves for cooked hams}

In Study 1, the temporal profiles differed among samples and the perceptions of the sensory sensations elicited by the cooked ham were not strongly time-dependent (Supplementary data, Fig. 2a). The samples were characterized by one or two attributes that remained the same over most of the consumption period. HamA was mainly characterized by the attributes soft and juicy during most of the consumption time, with higher citation proportions than for the average of the rest of samples. In contrast, hardness was the sensation used to describe HamB. Fibrous and smoked flavour were the most representative attributes in the sensory perception of HamC. For HamD, the attribute curves were quite flat, with no salient attributes, while for HamE the sensation of softness was perceived and ham flavour was also prominent.

In general, the longer fading time resulted in curves with higher citation proportions in which sample discrimination lasted longer (Fig. 1). However, small exceptions were detected, especially in the later stages of evaluation. The number of seconds in which juicy was significantly more cited in HamA than in other samples was higher when the fading time was short (22 s) than when the fading time was long (15 s).

\subsubsection{TCATA curves of strawberry-flavoured fresh cheese desserts}

In Study 2, the most relevant terms for describing the samples were creamy, smooth, sour, strawberry flavour and sweet (Supplementary data, Fig. 2b). All samples were perceived as creamy at the beginning, with this attribute reaching its maximum citation proportions at $5 \mathrm{~s}$. Dess A and DessC were also perceived as sweet, smooth and with strawberry flavour, which presented their maximum citation proportions from $5 \mathrm{~s}$ to 10 
s. In contrast, for the high-protein fat-free cheese products (samples DessB and DessD) the citation of sweet did not differ from the average for the rest of the samples. DessB also presented significantly higher citations of sour throughout the consumption time and of consistent at the beginning, progressing to roughness from $12 \mathrm{~s}$. As with the ham samples, the longer fading time resulted in curves with higher citation proportions and a greater number of seconds with significant differences between samples (Fig. 2). For example, in DessA and DessC only the longer fading time revealed significant differences compared to the other samples, in creaminess and smoothness, respectively. It should be noted that smoothness in sample DessA was significantly higher than in the rest of the samples at the beginning in the short fading test, whereas in the longer fading test the significant difference appeared almost at the end.Small exceptions in sample discrimination were also detected. For example, in the case of DessD the attributes roughness and mouth coating were significantly higher for this sample than for the others for a few seconds in the shorter fading scenario but not in the longer fading curves.

\subsubsection{TCATA curves for orange juice drinks}

In Study 3, the term most cited was artificial flavour, except for OrangA, which presented a higher citation proportion than the other samples for term sour (Supplementary data, Fig. 2c). Bitterness was prominent for OrangD, probably conferred by the sweetener acesulfame $\mathrm{K}$, which is associated with a bitter aftertaste (Schiffman, Booth, Losee, Pecore, \& Warwick, 1995). In general, compared to the other two studies, the attribute citation proportions and the number of seconds with significant differences between samples were both lower in Study 3 (Fig. 3). However, as in the other two studies, higher citation proportions were generally obtained for the longer fading time, such as orange flavour and bitter for sample OrangA or artificial flavour and orange flavour for sample OrangB. In contrast, with the shorter fading time the term bitter showed higher citation proportions and greater differences in OrangD than in the other samples in the first 10 seconds of evaluation.

\subsubsection{Sample discrimination in tests with different fading times}

For each attribute, the sum of the significant differences found for each sample relative to the average for the rest of the samples was calculated to compare sample discrimination between the fading duration tests (Tables 4, 5, and 6). Study 1 showed 
higher attribute discrimination in general among the samples, probably because the assessors were semi-trained panellists or because there were greater differences between samples. In Studies 2 and 3, where the assessors were consumers, the number of significant differences was lower, particularly in Study 3 where the sensory differences between samples were low. As already observed for the curves in general, more significant differences were observed in the long fading tests. However, the magnitude of this effect depended on the study and attribute. The biggest difference was found in Study 1, but only for the attributes soft, fibrous and juicy. In Study 2 the differences between the long fading and short fading tests were slight and were relevant only for the attributes sour and sweet. In Study 3, the number of seconds with significant differences was low for most attributes and the differences between short and long fading times were only relevant for sour.

\subsubsection{Differences in citation proportions between tests with different fading times}

As observed in the curves, at all times, for all the samples and all the studies, the citation proportions were higher for the long fading duration test. The number of seconds for which the citation proportions differed significantly between the short fading test and long fading test are shown in tables 4, 5, and 6. In general, the morecited terms tended to be those that showed higher differences in citation proportions between fading times. For instance, in samples HamA and HamB (Study 1) the highest differences were found in the terms soft and hard respectively; in Study 2 they were found in sample DessA and DessC for the highly-cited terms creamy and strawberry flavour and in sample DessB for sour; and in Study 3 they were observed in sample OrangB for the terms orange flavour and sweet.

\subsection{Sensory trajectories}

Correspondence analysis plots showing the trajectories obtained for the short and long fading tests are shown in Figures 4, 5 and 6 for Study 1, 2 and 3, respectively. The two dimensions explained more than $75 \%$ of the total inertia for all the studies.

In Study 1, the first factor mainly explained differences among samples, while differences with time were mainly explained by the second factor. The trajectory of each sample mostly started at the top of the map, with higher citation proportions of texture sensations (soft for HamA, and HamE, hard for HamB and fibrous for HamC), 
progressing to flavour attributes at the end (ham flavour and salty for sample HamA, HamB, and HamD, and smoked flavour for samples HamB and HamC).

In Study 2, the high-protein fat-free cheese products (DessB and DessD) were located to the right of the first dimension, especially in the case of sample DessB, which was placed near the sensation consistent at the beginning and progressed to the terms sour and roughness. In contrast, the regular version samples with a higher fat content and lower protein content (DessA and DessC) were located on the left side, progressing from the sensation creamy to smooth, strawberry flavour and sweet in the case of DessA, and to the terms, sweet and cheese flavour in the case of Dess C. In general, the samples presented long linear trajectories, which evolved in relation to the second dimension of the plot.

In Study 3, the first factor mainly explained differences in sour and sweet sensations. Samples OrangA and OrangeC differed especially at the beginning, being perceived as sour and sweet respectively. In general, all the samples were placed near to orange flavour and artificial, which were the most-cited terms. Sample OrangD evolved to bitterness, especially in the shorter fading time. All samples were located near each other but far from attributes, as the differences between samples were low.

In all three studies, the trajectory plots did not differ much between the tests with different fading durations and revealed similar information. However, the trajectories tended to be shorter and straighter for the long fading tests.

\section{Discussion}

The present work analyses the effect of different fading durations on how the TCATA evaluation task is performed and on the dynamic curves and trajectories.

The task took longer for the tests with long fading than for those with short fading time. This difference might indicate that when using a long fading time the evaluation was slower and finished later. However, it seemed probable that the difference came from cases where the assessors waited to press the stop button until all the attributes appeared unselected. On inspecting the data, the number of cases where the task was stopped with no attributes selected was higher in tests with short fading $(75 \%, 41 \%$ and $53 \%$ of cases in Studies 1, 2 and 3, respectively) than in the long fading tests (52\% 18\% and 23\%), 
which seems to indicate that none of the assessors systematically tended to wait until the selection of attributes had stopped.

For all three studies, the attributes remained selected significantly longer in the tests with a long fading time than in those with short fading and the participants selected them the same number of times on average regardless of the fading duration (Table 2). On the one hand it may indicate that in most cases the sensations lasted no longer than the short fading time employed ( 4 or $3 \mathrm{~s}$ ), so using the long ( 8 or $6 \mathrm{~s}$ ) fading time would prolong a sensation that in fact lasts less time than the fading period, thus overestimating the duration of the sensation and the significant differences between samples. On the other hand, it could indicate that the short fading time was too short and that some sensations that in fact applied remained unselected because the assessor did not have time to reselect them.

It is also important to note that overlaps (reselection of an attribute while still fading) were observed throughout the fading duration of the attributes, not only in the final seconds of fading, indicating that the participants did not wait until the fading finished and just selected and reselected the attributes as they perceived them. In the present work, the participants were instructed to select all the attributes that described the sensations they perceived and were told to check/make sure at every moment of the evaluation that the sensations they perceived appeared selected on the screen, as the terms would be automatically deselected. They did not receive any specific instruction about waiting until the attribute they still perceived was completely deselected before reselecting it. The idea was not to keep participants focused on the attributes' fading state, but only on checking the attributes, as the fading version of TCATA was in fact proposed to avoid the double task of selection and deselection in the original TCATA. Reselection of still-fading attributes occurred in all these three studies, indicating that most participants intuitively understood the task. For all the studies, the percentage of cases with overlaps was higher in general for the long fading tests. On the other hand, the presence of short gaps indicated that reselection of an attribute that still applied occurred after the button had faded to an unselected state, probably because the assessor was too busy to re-select it. The effect of fading time on the percentage of cases with gaps depended on the study and the attribute. Study 3 was the only one in which a longer fading time resulted in a noticeable reduction in the percentage of gaps for all the attributes. The predefined short fading time of $3 \mathrm{~s}$ showed a high number of gaps, which 
decreased drastically when using the $6 \mathrm{~s}$ fading duration. This would seem to indicate that $3 \mathrm{~s}$ was probably a too short fading time.

TCATA curves obtained using short and long fading times revealed very similar information on differences among the dynamic profiles of the samples. However, the longer fading times resulted in curves with higher citation proportions in which the significant differences between samples lasted longer. In all three studies, the effect of higher sample discrimination with a longer fading time appeared to be more noticeable in the most-cited or more relevant terms. The effect of fading duration was low in Studies 2 and 3, where the citation proportions were lower than in Study 1, probably due to there being less agreement on attribute selection, or due to time, or because they were conducted with consumers, or because the sensations changed faster with semisolid and liquid products. Vidal et.al (2017) proposed an alternative analysis for improving TCATA curves obtained with the fading version, which consists of detecting the small gaps between successive selections and filling them in (inputting them as selected). These authors showed that filling the gaps provided TCATA curves with higher citation proportions and increased the duration of the significant differences among samples, especially for the most relevant attributes for describing each sample. These effects seem to be the same ones as observed in the present study when using a longer fading time, indicating that filling the gaps and using a longer fading time could have similar effects on TCATA Fading curves. The gap filling procedure was conducted with data from this study. Gaps of a short duration $(<4 \mathrm{~s})$ were filled in and TCATA curves were constructed. As an example, a comparison of TCATA curves with and without gap filling for the short and long fading times of sample A of each study may be found in Supplementary data, Fig 3. As shown by the plot, gap filling resulted in TCATA curves with higher citation proportions than in the raw data curves. However, compared to the effect of using a longer fading time, the increase in citation that resulted from filling the gaps was very low. The average differences in citation proportion between the gap filling data curves and those constructed from the raw data were $11.8 \%$ vs $7.25 \%$ (Study 1), $8.8 \%$ vs $5.9 \%$ (Study 2), and $11.4 \%$ vs $8.6 \%$ (Study 3), for the short and long fading times, respectively. The increase in citations was slightly higher for the short fading time tests, as expected given the higher number of gaps they presented compared to the long fading times.

Regarding TCATA trajectories, correspondence analysis plots obtained from the long and short fading tests provided similar information on the evolution of sample 
trajectories, but it was observed that they tended to be shorter and straighter for the longer fading time. This effect may result from the fact that the increase in citation proportions when the fading duration increased affected the most relevant attributes selectively, enlarging the difference between them and the rest of attributes and resulting in a more extreme or "clear" profile. For the short fading tests, where the weight of less relevant attributes was higher, the trajectories described were more changeable and more difficult to interpret.

Overall, the results indicated that using a longer fading time could be more advisable to avoid delays in selecting successive attributes and the presence of gaps in temporal profiles. However, a longer fading time seemed to result in a possible overestimation of the duration of sensations, which could be especially critical for short or fast-changing sensations. Ideally, the fading duration should be long enough to give the assessor time to reselect an attribute (thus avoiding gaps) and short enough not to exceed the duration of the sensation. However, making this decision is not straightforward and no general rules can be given a priori because, as shown in the present study, the impact of fading length on the duration of attribute selection, number of gaps, and overlaps is studydependent and may vary with the number of attributes to be evaluated, the sample characteristics and the attribute considered. A pilot study with different fading durations would help to decide the shorter period that assessors find easiest, generating a "reasonable" number of gaps. The present study also showed that when using different fading durations in a "reasonable" range, the TCATA curves and TCATA trajectories obtained provide similar information, which makes the selection of fading duration less critical. Furthermore, using the gap filling approach proposed by Vidal et al. (2017) for data analysis compensates in part for selection of a too short fading duration, so in these cases filling in short gaps could be more advisable than using a fading time that might be too long. Another option would be to use predefined fading times adapted to the evaluation period and to the attribute. For example, a longer fading time at the beginning of the evaluation when the assessor is busiest (and a higher number of gaps is usually detected) and a shorter fading period for the rest of the evaluation (to avoid overestimation); or, as proposed by Ares et al. (2016), to use different fading times for different attributes. These adapted options would require a good knowledge of how sensations are perceived and could risk biasing the results.

\section{Conclusion}


When using the TCATA Fading method, the duration of fading significantly affects individual temporal profiles. Using a long fading duration is better for avoiding gaps in temporal profiles but it seems that it can overestimate the duration of short-lasting sensations. When using a longer fading duration, TCATA curves present higher citation proportions and the significant differences among samples last longer for terms that are more relevant. However, TCATA curves and trajectories obtained from tests with different fading durations provide very similar information about the dynamic sensory characteristics of samples.

\section{Acknowledgements}

The authors wish to thank the Spanish Ministry of the Economy and Competitiveness for financial support (project AGL-2016-75403-R) and for the Ramon y Cajal contract of author Tarrega (with the support of EU FEDER funds), the Generalitat Valenciana (Project Prometeo 2017/189). Mary Georgina Hardinge provided English language editing assistance.

\section{References}

Alcaire, F., Antúnez, L., Vidal, L., Zorn, S., Giménez, A., Castura, J. C., \& Ares, G. (2017). Comparison of static and dynamic sensory product characterizations based on check-all-that-apply questions with consumers. Food Research International, 97, 215-222.

Ares, G., Alcaire, F., Antúnez, L., Vidal, L., Giménez, A., \& Castura, J. C. (2017). Identification of drivers of (dis)liking based on dynamic sensory profiles: Comparison of Temporal Dominance of Sensations and Temporal Check-allthat-apply. Food Research International, 92, 79-87.

Ares, G., Castura, J. C., Antúnez, L., Vidal, L., Giménez, A., Coste, B., et al. (2016). Comparison of two TCATA variants for dynamic sensory characterization of food products. Food Quality and Preference, 54, 160-172. 
Ares, G., Jaeger, S. R., Antúnez, L., Vidal, L., Giménez, A., Coste, B., et al. (2015). Comparison of TCATA and TDS for dynamic sensory characterization of food products. Food Research International, 78, 148-158.

Baker, A. K., Castura, J. C., \& Ross, C. F. (2016). Temporal Check-All-That-Apply Characterization of Syrah Wine. Journal of Food Science, 81(6), S1521-S1529.

Boinbaser, L., Parente, M. E., Castura, J. C., \& Ares, G. (2015). Dynamic sensory characterization of cosmetic creams during application using Temporal CheckAll-That-Apply (TCATA) questions. Food Quality and Preference, 45, 33-40.

Castura, J. C. (2016). tempR: Temporal Sensory Data Analysis. R package version 0.9.9.10. http://www.cran.r-project.org/package=tempR/.

Castura, J. C., Antúnez, L., Giménez, A., \& Ares, G. (2016). Temporal Check-All-ThatApply (TCATA): A novel dynamic method for characterizing products. Food Quality and Preference, 47, 79-90.

Di Monaco, R., Su, C., Masi, P., \& Cavella, S. (2014). Temporal dominance of sensations: A review. Trends in Food Science and Technology, 38(2), 104-112.

Esmerino, E. A., Castura, J. C., Ferraz, J. P., Tavares Filho, E. R., Silva, R., Cruz, A. G., Freitas, M., Bolini, H. M. A. (2017). Dynamic profiling of different ready-todrink fermented dairy products: A comparative study using Temporal Check-AllThat-Apply (TCATA), Temporal Dominance of Sensations (TDS) and Progressive Profile (PP). Food Research International, 101, 249-258.

Fisher, R. A. (1935). The logic of inductive inference. Journal of the Royal Statistical Society, 98, 39-54.

Irwin, J. O. (1935). Tests of significance for differences between percentages based on small numbers. Metron, 12, 83-94.

ISO (2007). Sensory analysis: General guidance for the design of test rooms. ISO standard 8589. Geneva, Switzerland: International Organization for Standardization.

Jaeger, S. R., Alcaire, F., Hunter, D. C., Jin, D., Castura, J. C., \& Ares, G. (2018). Number of terms to use in temporal check-all-that-apply studies (TCATA and TCATA Fading) for sensory product characterization by consumers. Food Quality and Preference, 64, 154-159.

Jaeger, S. R., Beresford, M. K., Hunter, D. C., Alcaire, F., Castura, J. C., \& Ares, G. (2017). Does a familiarization step influence results from a TCATA task? Food Quality and Preference, 55, 91-97. 
McMahon, K. M., Culver, C., Castura, J. C., \& Ross, C. F. (2017). Perception of carbonation in sparkling wines using descriptive analysis (DA) and temporal check-all-that-apply (TCATA). Food Quality and Preference, 59, 14-26.

Meillon, S., Urbano, C., \& Schlich, P. (2009). Contribution of the Temporal Dominance of Sensations (TDS) method to the sensory description of subtle differences in partially dealcoholized red wines. Food Quality and Preference, 20(7), 490-499.

Nguyen, Q. C., Næs, T., \& Varela, P. (2018). When the choice of the temporal method does make a difference: TCATA, TDS and TDS by modality for characterizing semi-solid foods. Food Quality and Preference, 66, 95-106.

Oliveira, D., Antúnez, L., Giménez, A., Castura, J. C., Deliza, R., \& Ares, G. (2015). Sugar reduction in probiotic chocolate-flavored milk: Impact on dynamic sensory profile and liking. Food Research International, 75, 148-156.

Pineau, N., Cordelle, S., \& Schlich, P. (2003). Temporal dominance of sensations: A new technique to record several sensory attributes simultaneously over time. In 5th Pangborn Sensory Science Symposium. July 20-24. Boston, MA, USA (pp. 121).

Pineau, N., Schlich, P., Cordelle, S., Mathonnière, C., Issanchou, S., Imbert, A., et al. (2009). Temporal Dominance of Sensations: Construction of the TDS curves and comparison with time-intensity. Food Quality and Preference, 20(6), 450-455.

Ramsey, I., Ross, C., Ford, R., Fisk, I., Yang, Q., Gomez-Lopez, J., \& Hort, J. (2018). Using a combined temporal approach to evaluate the influence of ethanol concentration on liking and sensory attributes of lager beer. Food Quality and Preference, 68, 292-303.

Rizo, A., Peña, E., Alarcon-Rojo, A. D., Fiszman, S., \& Tarrega, A. (2018). Relating texture perception of cooked ham to the bolus evolution in the mouth. Food Research International (in press).

Schiffman, S. S., Booth, B. J., Losee, M. L., Pecore, S. D., \& Warwick, Z. S. (1995). Bitterness of sweeteners as a function of concentration. Brain Research Bulletin, $36(5), 505-513$.

Schlich, P. (2017). Temporal Dominance of Sensations (TDS): a new deal for temporal sensory analysis. Current Opinion in Food Science, 15, 38-42.

Sokolowsky, M., \& Fischer, U. (2012). Evaluation of bitterness in white wine applying descriptive analysis, time-intensity analysis, and temporal dominance of sensations analysis. Analytica Chimica Acta, 732, 46-52. 
Thomas, A., Visalli, M., Cordelle, S., \& Schlich, P. (2015). Temporal Drivers of Liking. Food Quality and Preference, 40(PB), 365-375.

Varela, P., Pintor, A., \& Fiszman, S. (2014). How hydrocolloids affect the temporal oral perception of ice cream. Food Hydrocolloids, 36, 220-228.

Vidal, L., Castura, J. C., Coste, B., Picallo, A., Jaeger, S. R., \& Ares, G. (2017). Analysis of TCATA Fading data: Imputation of gaps in temporal profiles. Food Quality and Preference, 59, 114-12 
Table 1. Summary of the characteristics of the three studies included in this work

\begin{tabular}{|c|c|c|c|c|c|c|c|c|}
\hline Study & Products & $\begin{array}{l}\text { Type of } \\
\text { assessor }\end{array}$ & $\begin{array}{c}\text { Number } \\
\text { of } \\
\text { assessors } \\
\end{array}$ & Replicates & $\begin{array}{c}\text { Task } \\
\text { duration } \\
(\mathbf{s}) \\
\end{array}$ & $\begin{array}{c}\mathrm{N}^{\mathbf{o}} \text { of } \\
\text { attributes }\end{array}$ & List of attributes & $\begin{array}{c}\text { Fading } \\
\text { duration } \\
(\mathrm{s}) \\
\end{array}$ \\
\hline 1 & $\begin{array}{c}\text { Cooked } \\
\text { ham }\end{array}$ & $\begin{array}{l}\text { Semi- } \\
\text { trained }\end{array}$ & 17 & 3 & 40 & 7 & $\begin{array}{l}\text { soft, hard, fibrous, } \\
\text { juicy, ham flavour, } \\
\text { smoked flavour, } \\
\text { salty }\end{array}$ & and 8 \\
\hline 2 & $\begin{array}{c}\text { Strawberry } \\
\text { dessert }\end{array}$ & Consumers & 68 & 1 & 90 & 9 & $\begin{array}{l}\text { roughness, cheese } \\
\text { flavour, consistent, } \\
\text { creamy, mouth } \\
\text { coating, smooth, } \\
\text { sour, strawberry } \\
\text { flavour, sweet }\end{array}$ & 4 and 8 \\
\hline 3 & $\begin{array}{l}\text { Orange } \\
\text { juice }\end{array}$ & Consumers & 70 & 1 & 60 & 5 & $\begin{array}{l}\text { artificial flavour, } \\
\text { bitter, orange, sour, } \\
\text { sweet }\end{array}$ & 3 and 6 \\
\hline
\end{tabular}


Table 2. Average duration and number of selections of each attribute during TCATA fading evaluation of cooked ham, strawberry dessert and orange juice (Studies 1, 2 and 3 , respectively), using two different fading durations. Only the cases where the attribute was selected were included in the calculations. Effect of fading time and sample according to ANOVA tests ( $\mathrm{p}$-value).

\begin{tabular}{|c|c|c|c|c|c|c|c|c|}
\hline & \multicolumn{4}{|c|}{$\begin{array}{l}\text { DURATION OF ATTRIBUTE } \\
\text { SELECTION (s) }\end{array}$} & \multicolumn{4}{|c|}{$\begin{array}{l}\text { NUMBER OF } \\
\text { SELECTIONS }\end{array}$} \\
\hline \multicolumn{9}{|l|}{ STUDY 1} \\
\hline \multirow[t]{2}{*}{ Attributes } & $\begin{array}{c}4 \mathrm{~s} \\
\text { fading }\end{array}$ & $\begin{array}{c}8 \mathrm{~s} \\
\text { fading }\end{array}$ & \multicolumn{2}{|c|}{ p-value } & $\begin{array}{c}4 \mathrm{~s} \\
\text { fading }\end{array}$ & $\begin{array}{c}8 \mathrm{~s} \\
\text { fading }\end{array}$ & \multicolumn{2}{|c|}{ p-value } \\
\hline & & & $\begin{array}{l}\text { Fading } \\
\text { time }\end{array}$ & Sample & & & $\begin{array}{l}\text { Fading } \\
\text { time }\end{array}$ & Sample \\
\hline Soft & 11.1 & 17.3 & $<0.001$ & 0.002 & 3.0 & 2.6 & 0.161 & 0.025 \\
\hline Hard & 8.1 & 15.3 & $<0.001$ & 0.001 & 2.2 & 2.5 & 0.295 & 0.001 \\
\hline Fibrous & 9.5 & 16.7 & $<0.001$ & 0.001 & 2.5 & 2.5 & 0.993 & 0.001 \\
\hline Juicy & 7.9 & 14.3 & $<0.001$ & 0.001 & 2.1 & 2.0 & 0.676 & 0.003 \\
\hline Ham flavour & 9.1 & 14.6 & $<0.001$ & 0.099 & 2.5 & 2.1 & 0.105 & 0.062 \\
\hline Smoked flavour & 11.1 & 16.7 & $<0.001$ & 0.002 & 3.2 & 2.6 & 0.116 & 0.015 \\
\hline Salty & 10.3 & 16.0 & $<0.001$ & 0.007 & 2.8 & 2.5 & 0.357 & 0.134 \\
\hline \multicolumn{9}{|l|}{ STUDY 2} \\
\hline \multirow[t]{2}{*}{ Attributes } & $\begin{array}{c}4 \mathrm{~s} \\
\text { fading }\end{array}$ & $\begin{array}{c}8 \mathrm{~s} \\
\text { fading }\end{array}$ & \multicolumn{2}{|c|}{$p$-value } & $\begin{array}{c}4 \mathrm{~s} \\
\text { fading }\end{array}$ & $\begin{array}{c}8 \mathrm{~s} \\
\text { fading }\end{array}$ & \multicolumn{2}{|c|}{ p-value } \\
\hline & & & $\begin{array}{c}\text { Fading } \\
\text { time }\end{array}$ & Sample & & & $\begin{array}{c}\text { Fading } \\
\text { time }\end{array}$ & Sample \\
\hline Roughness & 5.3 & 10.1 & $<0.001$ & 0.841 & 1.5 & 1.8 & 0.124 & 0.208 \\
\hline Cheese flavour & 5.6 & 10.4 & $<0.001$ & 0.373 & 1.6 & 1.7 & 0.575 & 0.055 \\
\hline Consistent & 5.7 & 9.5 & $<0.001$ & 0.627 & 1.9 & 1.4 & 0.043 & 0.985 \\
\hline Creamy & 5.6 & 9.9 & $<0.001$ & 0.582 & 1.7 & 1.5 & 0.260 & 0.850 \\
\hline Mouth coating & 5.3 & 9.9 & $<0.001$ & 0.492 & 1.7 & 1.6 & 0.735 & 0.444 \\
\hline Smooth & 5.6 & 9.1 & $<0.001$ & 0.176 & 1.6 & 1.4 & 0.124 & 0.292 \\
\hline Sour & 7.0 & 13.0 & $<0.001$ & $<0.001$ & 2.2 & 2.6 & 0.918 & 0.234 \\
\hline $\begin{array}{l}\text { Strawberry } \\
\text { flavour }\end{array}$ & 6.9 & 10.9 & $<0.001$ & 0.286 & 2.2 & 1.9 & 0.140 & 0.307 \\
\hline Sweet & 7.3 & 12.5 & $<0.001$ & 0.819 & 2.2 & 2.1 & 0.499 & 0.541 \\
\hline \multicolumn{9}{|l|}{ STUDY 3} \\
\hline \multirow[t]{2}{*}{ Attributes } & $\begin{array}{c}\text { 3 s } \\
\text { fading }\end{array}$ & $\begin{array}{c}6 \mathrm{~s} \\
\text { fading }\end{array}$ & \multicolumn{2}{|c|}{ p-value } & $\begin{array}{c}3 \mathrm{~s} \\
\text { fading }\end{array}$ & $\begin{array}{c}6 \mathrm{~s} \\
\text { fading }\end{array}$ & \multicolumn{2}{|c|}{ p-value } \\
\hline & & & $\begin{array}{l}\text { Fading } \\
\text { time }\end{array}$ & Sample & & & $\begin{array}{c}\text { Fading } \\
\text { time }\end{array}$ & Sample \\
\hline Artificial flavour & 7.6 & 11.4 & $<0.001$ & 0.295 & 3.3 & 3.0 & 0.524 & 0.103 \\
\hline Bitter & 6.1 & 9.1 & $<0.001$ & 0.027 & 2.5 & 2.3 & 0.623 & 0.099 \\
\hline Orange flavour & 7.1 & 10.3 & $<0.001$ & 0.364 & 2.8 & 2.3 & 0.654 & 0.165 \\
\hline Sour & 6.1 & 9.4 & $<0.001$ & 0.023 & 2.3 & 2.1 & 0.178 & 0.025 \\
\hline Sweet & 5.8 & 10.5 & $<0.001$ & 0.297 & 2.2 & 2.4 & 0.465 & 0.021 \\
\hline
\end{tabular}


Table 3. Percentage of cases with successive selections of the same attribute out of total cases where the attribute was selected and percentage of cases with gaps $(<4 \mathrm{~s})$ and overlaps out of the total cases with successive selections, during TCATA fading evaluation of cooked ham, strawberry dessert and orange juice (Studies 1, 2 and 3, respectively) using two different fading durations.

\begin{tabular}{|c|c|c|c|c|c|c|}
\hline & $\begin{array}{r}\% 0 \\
\text { WITH } \\
\text { SELE } \\
\text { TH } \\
\text { AT } 1\end{array}$ & $\begin{array}{l}\text { SES* } \\
\text { ESIVE } \\
\text { NS OF } \\
\text { ME } \\
\text { JTE }\end{array}$ & \multicolumn{2}{|c|}{$\begin{array}{c}\% \text { OF CASES WITH } \\
\text { GAPS }(<4 \mathrm{~s})\end{array}$} & \multicolumn{2}{|c|}{$\begin{array}{c}\text { \% OF CASES WITH } \\
\text { OVERLAPPING }\end{array}$} \\
\hline $\begin{array}{l}\text { STUDY } 1 \\
\end{array}$ & Fadir & ation & \multicolumn{2}{|c|}{ Fading duration } & \multicolumn{2}{|c|}{ Fading duration } \\
\hline Attributes & $4 \mathrm{~s}$ & $8 \mathrm{~s}$ & $4 s$ & $8 \mathrm{~s}$ & $4 s$ & $8 \mathrm{~s}$ \\
\hline Soft & 68.0 & 64.6 & 47.6 & 41.5 & 33.5 & 71.8 \\
\hline Hard & 50.5 & 52.9 & 58.9 & 34.9 & 32.7 & 73.7 \\
\hline Fibrous & 61.2 & 63.2 & 50.5 & 40.4 & 24.7 & 58.3 \\
\hline Juicy & 56.8 & 52.9 & 38.7 & 42.0 & 20.5 & 49.4 \\
\hline Ham flavour & 59.5 & 58.3 & 42.2 & 37.9 & 26.1 & 50.0 \\
\hline Smoked flavour & 65.3 & 57.3 & 70.5 & 34.8 & 37.5 & 74.5 \\
\hline Salty & 59.9 & 61.4 & 54.0 & 38.3 & 38.8 & 66.0 \\
\hline \multicolumn{7}{|l|}{ STUDY 2} \\
\hline Attributes & $4 s$ & $8 \mathrm{~s}$ & $4 s$ & $8 \mathrm{~s}$ & $4 s$ & $8 \mathrm{~s}$ \\
\hline Roughness & 35.4 & 39.0 & 32.1 & 31.3 & 42.7 & 87.5 \\
\hline Cheese flavour & 30.2 & 38.9 & 27.2 & 27.0 & 47.8 & 67.6 \\
\hline Consistent & 31.6 & 22.4 & 33.5 & 9.10 & 69.8 & 85.5 \\
\hline Creamy & 32.1 & 29.2 & 39.0 & 26.2 & 52.0 & 67.2 \\
\hline Mouth coating & 27.8 & 27.4 & 16.7 & 34.5 & 59.8 & 62.1 \\
\hline Smooth & 30.8 & 23.9 & 17.8 & 24.3 & 53.2 & 64.9 \\
\hline Sour & 54.0 & 58.1 & 42.8 & 24.6 & 50.0 & 82.0 \\
\hline Strawberry flavour & 44.3 & 44.0 & 40.7 & 21.0 & 49.4 & 71.6 \\
\hline Sweet & 50.6 & 52.6 & 40.6 & 28.4 & 48.0 & 72.8 \\
\hline \multicolumn{7}{|l|}{ STUDY 3} \\
\hline Attributes & $3 \mathrm{~s}$ & $6 s$ & $3 \mathrm{~s}$ & $6 s$ & $3 \mathrm{~s}$ & $6 s$ \\
\hline Artificial flavour & 43.2 & 56.9 & 51.2 & 32.5 & 52.1 & 65.0 \\
\hline Bitter & 59.7 & 48.6 & 41.0 & 13.3 & 50.6 & 67.3 \\
\hline Orange flavour & 57.5 & 52.1 & 55.0 & 35.8 & 38.9 & 69.4 \\
\hline Sour & 52.7 & 57.4 & 58.0 & 38.2 & 39.8 & 64.2 \\
\hline Sweet & 49.4 & 56.4 & 48.3 & 35.6 & 42.5 & 69.3 \\
\hline
\end{tabular}

*Case: Each evaluation of the study (assessor $x$ replicate $x$ attribute $x$ sample) 
Table 4. Number of seconds for which the citation proportion of each attribute was significantly higher than the average of the rest of the samples for each fading time experiment (sample discrimination), and for which the citation proportion of each attribute differed significantly between the two fading durations (differences between short and long fading tests) in Study 1 (cooked ham).

\begin{tabular}{cccccccc}
\hline & \multicolumn{6}{c}{ SAMPLE } & \multicolumn{5}{c}{ DIFFERENCES BETWEEN SHORT AND } \\
& DISCRIMINATION & \multicolumn{5}{c}{ Samples } \\
\hline & \multicolumn{1}{c}{ Fading duration } & \multicolumn{5}{c}{ ONG FADING TESTS } \\
\hline Attributes & $\mathbf{4}$ s & $\mathbf{8 ~ s}$ & A & B & C & D & E \\
\hline Soft & 49 & 70 & 17 & 0 & 0 & 4 & 13 \\
Hard & 38 & 40 & 0 & 10 & 10 & 0 & 0 \\
Fibrous & 40 & 58 & 0 & 5 & 9 & 7 & 0 \\
Juicy & 25 & 33 & 1 & 9 & 0 & 20 & 22 \\
Ham flavour & 4 & 3 & 4 & 1 & 6 & 3 & 1 \\
Smoked flavour & 39 & 41 & 1 & 0 & 7 & 0 & 0 \\
Salty & 13 & 18 & 2 & 6 & 1 & 10 & 8 \\
\hline
\end{tabular}


Table 5. Number of seconds for which the citation proportion of each attribute was significantly higher than the average of the rest of the samples for each fading time experiment (sample discrimination), and for which the citation proportion of each attribute differed significantly between the two fading durations (differences between short and long fading tests) in Study 2 (strawberry dessert).

\begin{tabular}{lcccccc}
\hline & \multicolumn{2}{c}{$\begin{array}{c}\text { SAMPLE } \\
\text { DISCRIMINATION }\end{array}$} & \multicolumn{5}{c}{ DIFFERENCES BETWEEN SHORT } \\
AND LONG FADING TESTS \\
\hline \multicolumn{1}{c}{ Fading duration } & \multicolumn{5}{c}{ Samples } \\
\hline Roughness & $\mathbf{4}$ & $\mathbf{8}$ s & A & B & C & D \\
Cheese flavour & 7 & 10 & 7 & 6 & 0 & 0 \\
Consistent & 7 & 8 & 9 & 0 & 8 & 12 \\
Creamy & 1 & 5 & 14 & 8 & 20 & 10 \\
Mouth coating & 2 & 0 & 2 & 6 & 5 & 1 \\
Smooth & 7 & 14 & 15 & 0 & 6 & 8 \\
Sour & 25 & 37 & 0 & 23 & 4 & 1 \\
Strawberry flavour & 11 & 17 & 11 & 7 & 12 & 4 \\
Sweet & 7 & 21 & 17 & 0 & 15 & 15 \\
\hline
\end{tabular}


Table 6. Number of seconds for which the citation proportion of each attribute was significantly higher than the average of the rest of the samples for each fading time experiment (sample discrimination), and for which the citation proportion of each attribute differed significantly between the two fading durations (differences between short and long fading tests) in Study 3 (orange juice).

\begin{tabular}{lcccccc}
\hline & \multicolumn{2}{c}{ SAMPLE } & \multicolumn{3}{c}{ DIFFERENCES BETWEEN SHORT } \\
& DISCRIMINATION & \multicolumn{5}{c}{ AND LOMPles } \\
\hline \multicolumn{1}{c}{ Fading duration } & $\mathbf{6}$ & $\mathbf{A}$ & $\mathbf{B}$ & $\mathbf{C}$ & D \\
\hline Artificial flavour & 6 & 2 & 6 & 4 & 7 & 7 \\
Bitter & 8 & 12 & 3 & 0 & 0 & 0 \\
Orange flavour & 1 & 2 & 10 & 14 & 8 & 7 \\
Sour & 12 & 22 & 3 & 0 & 0 & 6 \\
Sweet & 8 & 11 & 2 & 12 & 7 & 14 \\
\hline
\end{tabular}



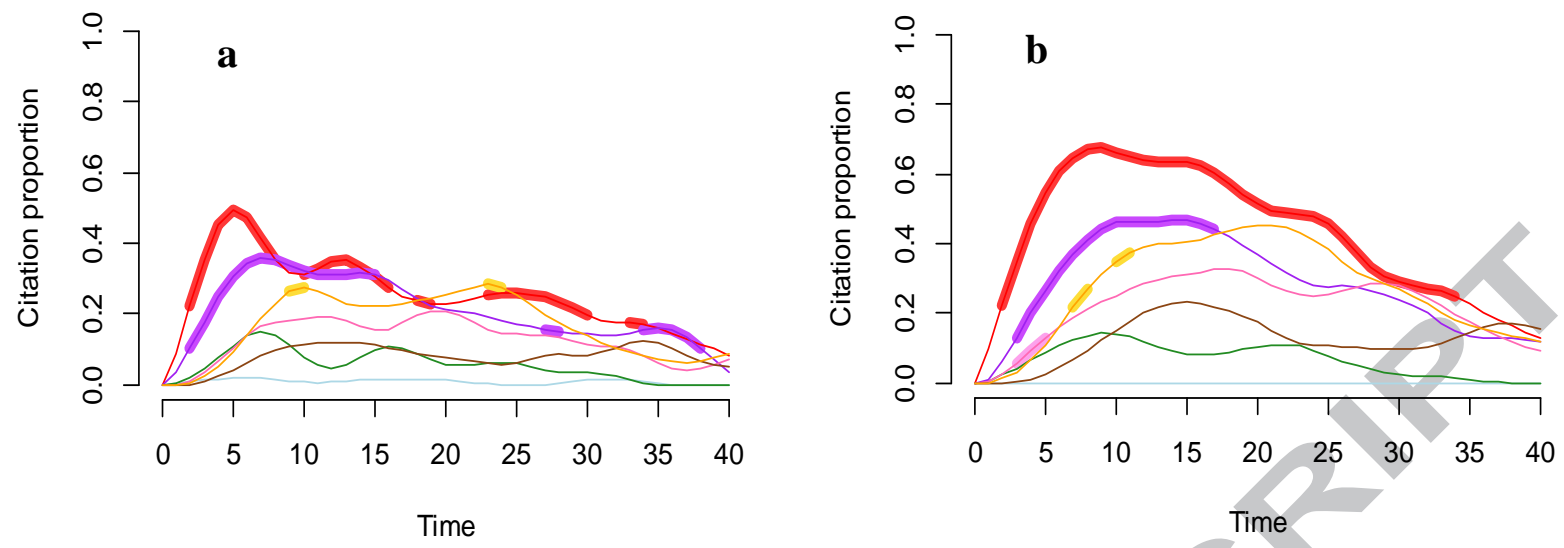

Fig. 1. TCATA curves of sample HamA of Study 1 using two fading times: 4 seconds (a) and 8 seconds (b). Attributes (soft - , hard - , juicy - , fibrous - , ham flavour - smoked flavour -, salty -). Highlighted sections indicate a significantly higher proportion of citations of the attribute at that evaluation time compared to the mean value for the rest of the samples in the same fading time category. Times expressed in seconds. 

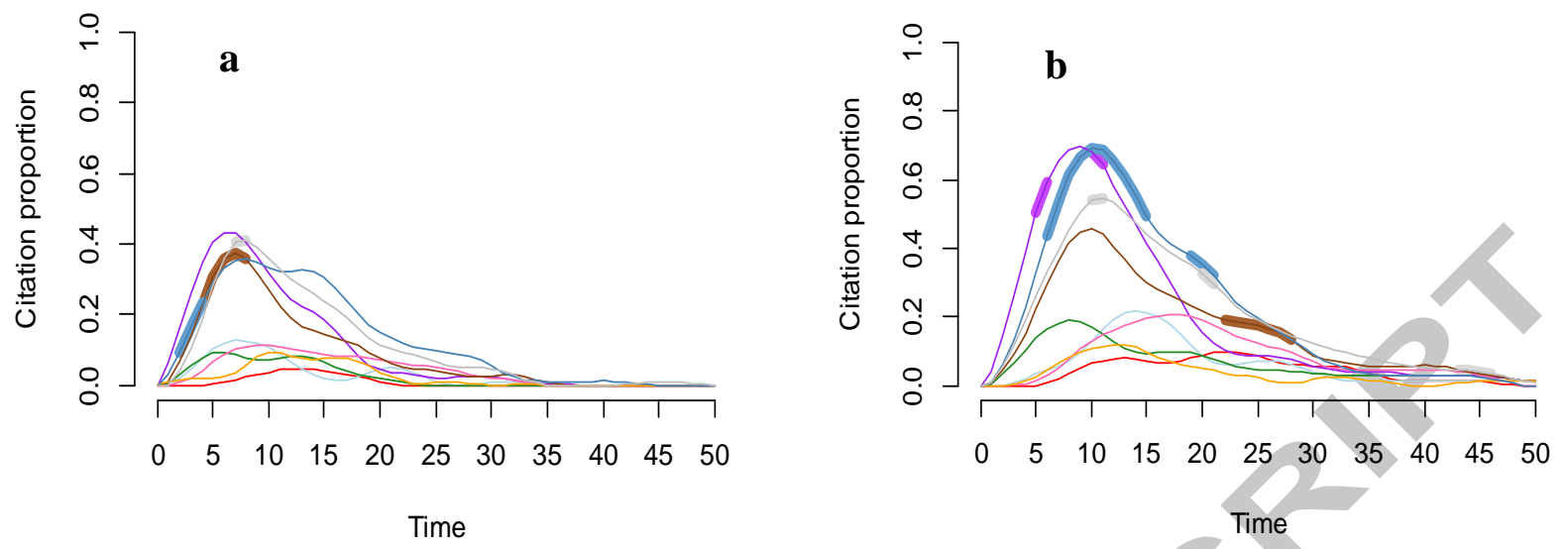

Fig. 2. TCATA curves of the sample DessA of Study 2 using two fading times: 4 seconds (a) and 8 seconds (b). Attributes (roughness - , cheese flavour - , strawberry flavour $\boldsymbol{-}$, creamy -, mouth coating - , sweet - , sour - , smooth - , and consistent -). Highlighted sections indicate a significantly higher proportion of citations of the attribute at that evaluation time compared to the mean value for the rest of the samples in the same fading time category. Times expressed in seconds. 

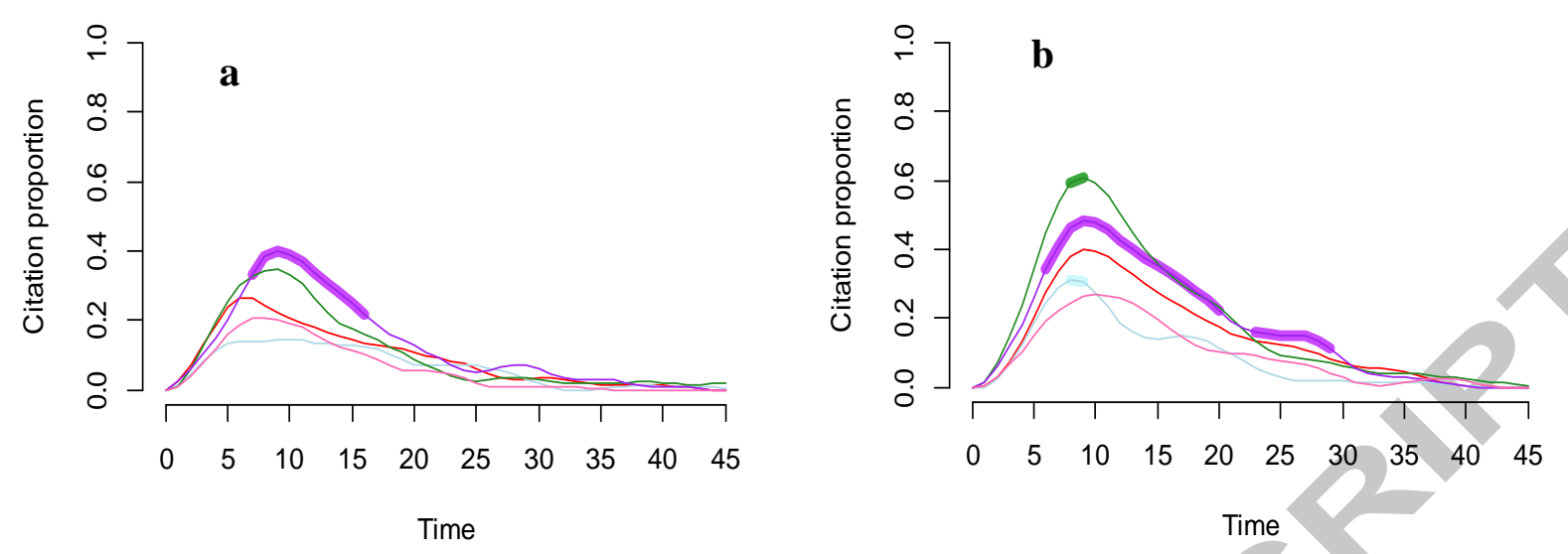

Fig. 3. TCATA curves of sample OrangA of Study 3 using two fading times: 3 seconds (a) and 6 seconds (b). Attributes (artificial flavour - , bitter - , orange flavour - , sweet - , sour -). Highlighted sections indicate a significantly higher proportion of citations of the attribute at that evaluation time compared to the mean value for the rest of the samples in the same fading time category. Times expressed in seconds. 


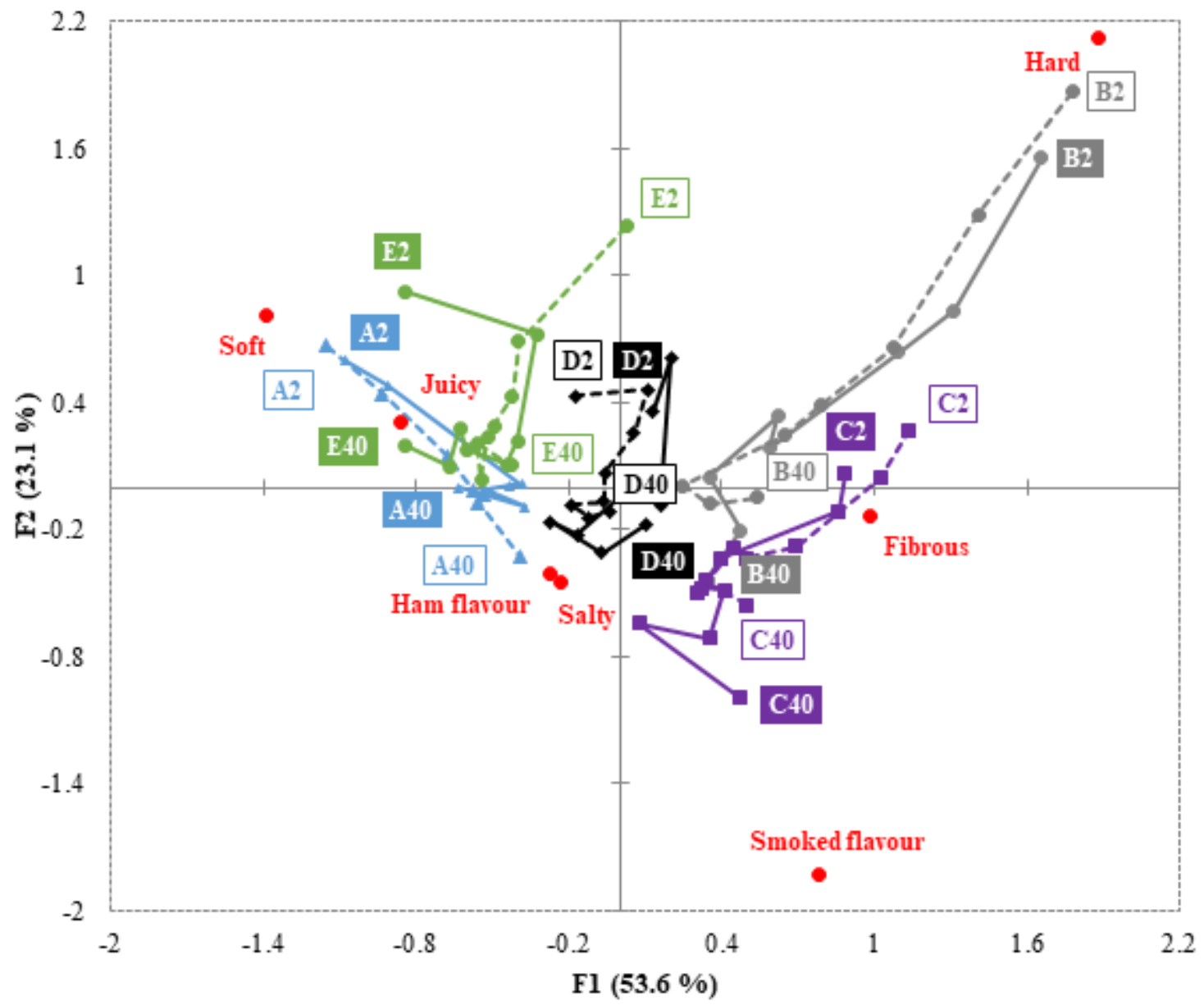

Fig. 4. Sensory trajectories of ham samples (A, B, C, D, and E) of Study 1 during its consumption from 2 to $40 \mathrm{~s}$ obtained through correspondence analysis of TCATA data with two different fading times: $4 \mathrm{~s}$ (continuous line) and $8 \mathrm{~s}$ (dotted line). 


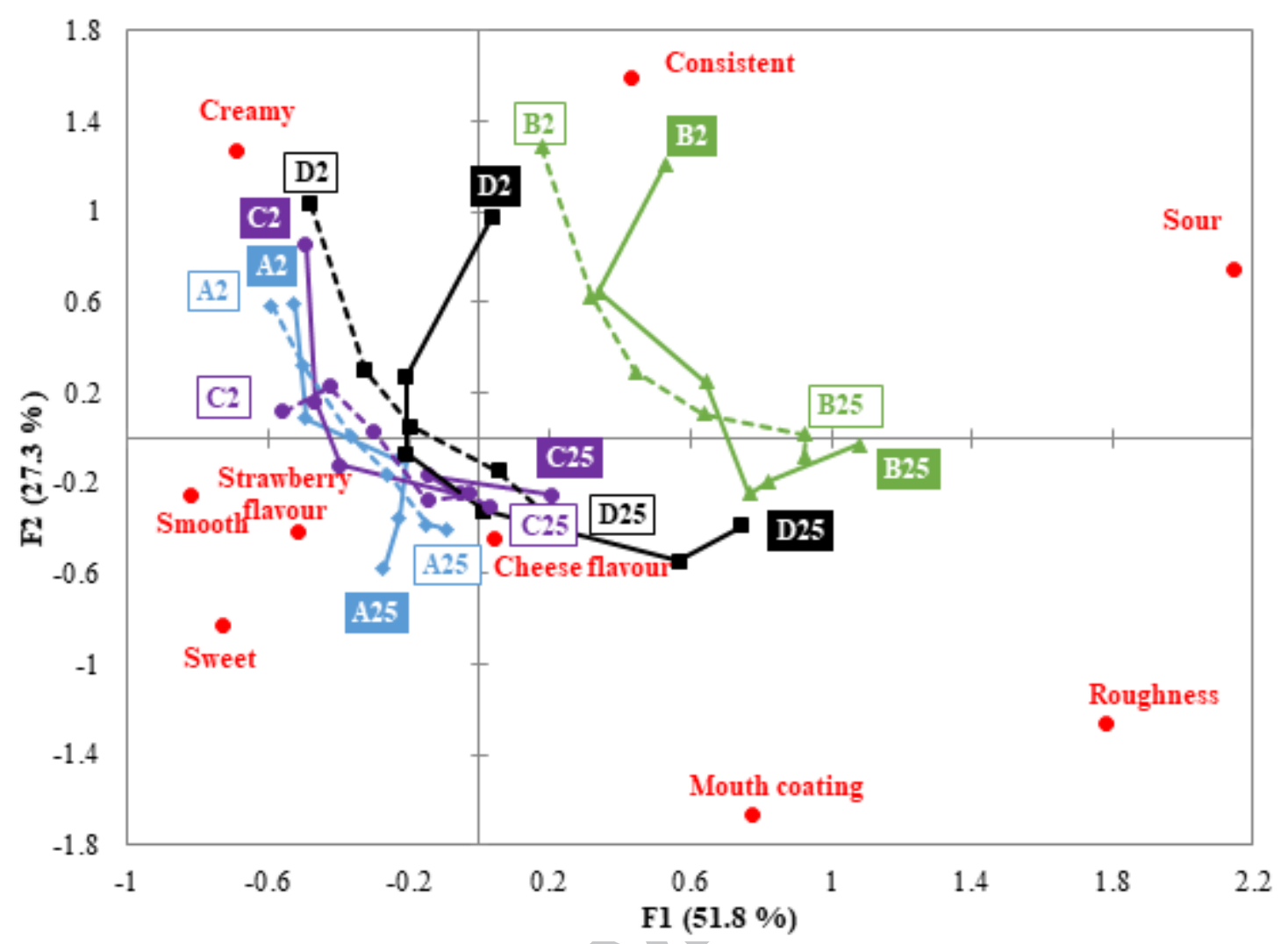

Fig. 5. Sensory trajectories of strawberry dessert samples (A, B, C, and D) of Study 2 during its consumption from 2 to $25 \mathrm{~s}$ obtained through correspondence analysis of TCATA data with two different fading times: $4 \mathrm{~s}$ (continuous line) and $8 \mathrm{~s}$ (dotted line). 


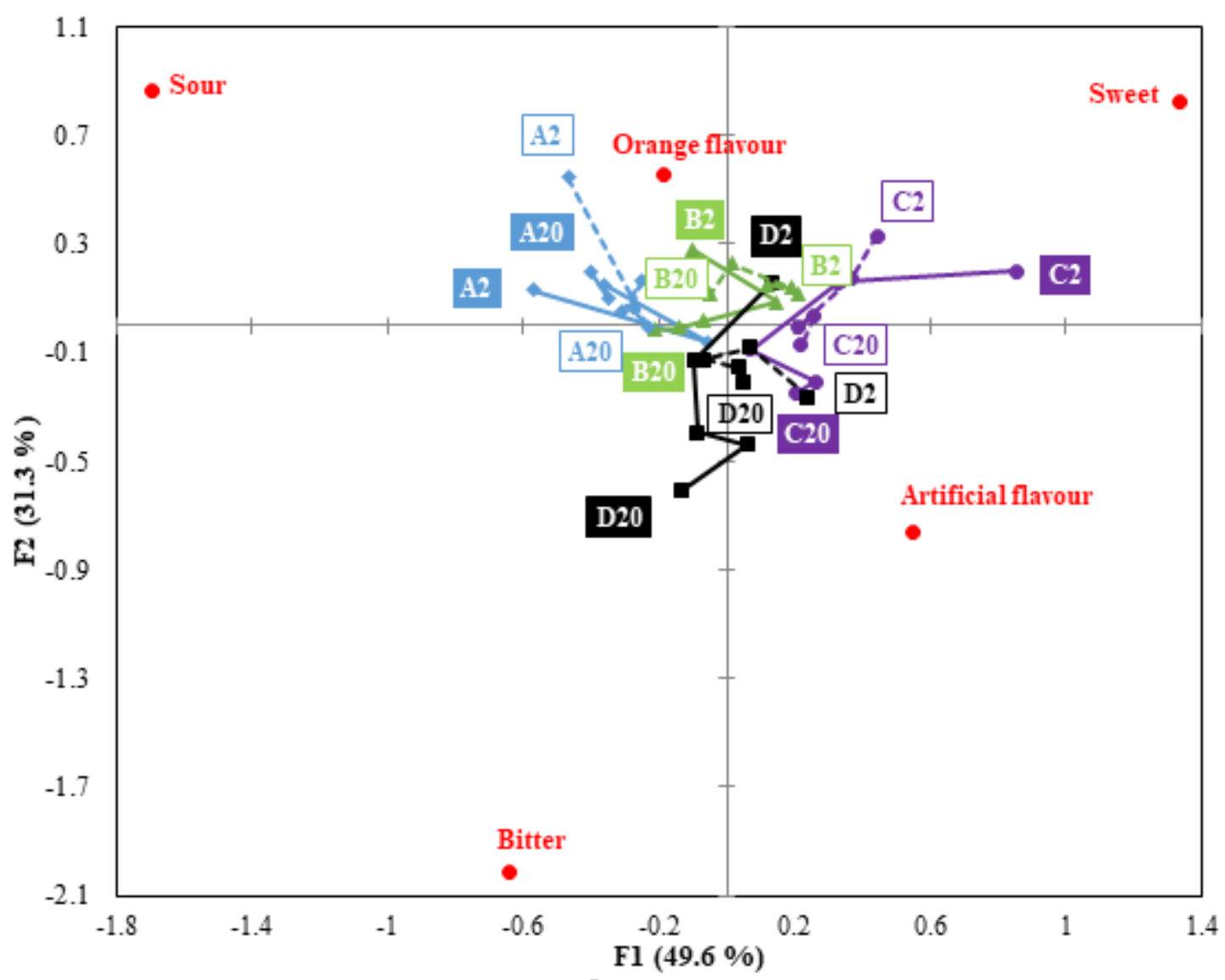

Fig. 6. Sensory trajectories of orange juice samples (A, B, C, and D) of Study 3 during its consumption from 2 to $20 \mathrm{~s}$ obtained through correspondence analysis of TCATA data with two different fading times: $3 \mathrm{~s}$ (continuous line) and $6 \mathrm{~s}$ (dotted line). 
Highlights

The influence of fading duration in TCATA was evaluated in three different studies.

The number of selections for an attribute did not much vary with the duration of fading.

A longer fading duration resulted in TCATA-curves with higher citation proportions.

Same information on differences in dynamic sensory perception of the products was obtained with different fading durations. 